The Astrophysical Journal, 680:32-40, 2008 June 10

(C) 2008. The American Astronomical Society. All rights reserved. Printed in U.S.A.

\title{
THE STELLAR INITIAL MASS FUNCTION AT THE EPOCH OF REIONIZATION
}

\author{
RANGA-RAM CHARY ${ }^{1}$ \\ Received 2007 December 6; accepted 2008 February 19
}

\begin{abstract}
I provide estimates of the ultraviolet and visible light luminosity density at $z \sim 6$ after accounting for the contribution from faint galaxies below the detection limit of deep HST and Spitzer surveys. I find that the rest-frame $V$-band luminosity density is a factor of $\sim 2-3$ below the ultraviolet luminosity density at $z \sim 6$. This implies that the maximal age of the stellar population at $z \sim 6$, for a Salpeter initial mass function (IMF) and a single, passively evolving burst, must be $\lesssim 100$ Myr. If the stars in $z \sim 6$ galaxies are remnants of the star formation that was responsible for ionizing the intergalactic medium, reionization must have been a brief process that was completed at $z<7$. This assumes the most current estimates of the clumping factor and escape fraction and a Salpeter slope extending up to $200 M_{\odot}$ for the stellar IMF $\left(d N / d M \propto M^{\alpha}, \alpha=-2.3\right)$. Unless the ratio of the clumping factor to escape fraction is less than 60 , a Salpeter slope for the stellar IMF and reionization redshift higher than 7 are ruled out. In order to maintain an ionized intergalactic medium from redshift 9 onward, the stellar IMF must have a slope of $\alpha=-1.65$ even if stars as massive as $\sim 200 M_{\odot}$ are formed. Correspondingly, if the intergalactic medium was ionized from redshift 11 onward, the IMF must have $\alpha \sim-1.5$. The range of stellar mass densities at $z \sim 6$ straddled by IMFs which result in reionization at $z>7$ is $(1.3 \pm 0.4) \times 10^{7} M_{\odot} \mathrm{Mpc}^{-3}$.
\end{abstract}

Subject headings: early universe — galaxies: high-redshift — galaxies: stellar content

\section{INTRODUCTION}

Deep optical/infrared imaging surveys with HST and Spitzer are providing an unprecedented opportunity to study star-forming galaxies at high redshifts. It is increasingly clear that these starforming galaxies are responsible for reionizing the intergalactic medium (IGM), a process which seems to be complete by $z \sim 6$ (Becker et al. 2001; Fan et al. 2006). The shape of the luminosity function of high-redshift active galactic nuclei detected by the Sloan Digital Sky Survey (SDSS) indicates that ionizing photons from supermassive black holes fall many orders of magnitude short of the minimum required to ionize the IGM (Fan et al. 2001). Even the current star formation in Lyman break galaxies (LBGs) at $z \sim 6$ as measured by Bouwens et al. $(2006,2007)$ falls about a factor of 6-9 below the minimum required to maintain an ionized IGM for canonical estimates of two key parameters, the clumping factor of the gas and the escape fraction, assuming a Salpeter initial mass function (IMF). One possibility is that the contribution from star formation in faint galaxies, below the detection threshold of current surveys, is higher than has been estimated. This translates to a faint-end slope for the galaxy ultraviolet luminosity function of -1.9 , a value that is considerably steeper than the value of -1.74 that has currently been measured (Bouwens et al. 2007). Evidence for such a steep slope is present in estimates of star formation rate densities derived from the comoving gamma-ray burst (GRB) number density (Chary et al. 2007a). However, that measurement has large uncertainties limited by the small number of high-redshift GRBs currently known and the uncertain conversion between GRB number density and star formation rate density.

An alternate possibility is that the star formation rate in galaxies currently detected at $z \sim 6$ was higher in the past. This implies that the ultraviolet (UV) luminosity function of galaxies must show luminosity evolution between $z \sim 6$ and higher red-

\footnotetext{
${ }^{1}$ Spitzer Science Center, California Institute of Technology, MS 220-6, Pasadena, CA 91125; rchary@caltech.edu.
}

shifts. Although constraints on the UV luminosity function at higher redshift are difficult to obtain from existing data, the upper limits on bright Lyman break galaxies at $z \gg 6$ seem to rule out such an evolution (Bouwens et al. 2004). In fact, the upper limits seem to suggest a negative evolution with increasing redshift, in the sense that galaxies at the bright end of the UV luminosity function at $z \sim 6$ were fainter at higher redshift in the UV. There are no available constraints on the evolution of the faint end of the UV luminosity function at $z>6$.

Measurement of the stellar mass density provides an alternate constraint on the past history of star formation. Deep Spitzer surveys which detect the rest-frame visible light from high-redshift galaxies allow the stellar mass in galaxies at $z \sim 6$ to be measured (Yan et al. 2006; Stark et al. 2007; Eyles et al. 2005). The galaxies which are detected in rest-frame optical light are primarily the brightest galaxies in the UV. The stellar mass density directly inferred from the Spitzer detections and stacking analysis is, therefore, a lower limit. By making reasonable assumptions for the rest-frame $V$-band luminosity of galaxies which are undetected in the Spitzer data but seen in the UV, it is possible to estimate the true comoving stellar mass density at $z \sim 6$. Since the stars at $z \sim 6$ are remnants of star formation at higher redshifts, we can use this stellar mass density estimate to constrain the number of ionizing photons produced by star formation in the past for comparison with the minimum number of photons required to keep the IGM ionized.

At present, the exact redshift of reionization is not known. Wilkinson Microwave Anisotropy Probe (WMAP) polarization measurements indicate a large optical depth to scattering of microwave background photons, suggesting that the IGM was ionized at some time between redshifts of 7 and 14 (Kogut et al. 2003; Spergel et al. 2007).

In this paper, we estimate the optical and UV luminosity density at $z \sim 6$ after correcting for faint galaxies undetected by current surveys. We then calculate the stellar mass density and average age of the stellar population in galaxies, required to reproduce the luminosity density estimates for different shapes of 
the stellar IMF. We also quantify the number of ionizing photons produced over the past history of the starburst and assess if they are sufficient to ionize the IGM at $z>6$. Finally, we constrain the stellar IMF at $z>6$ as a function of the reionization history of the universe. A standard $\Omega_{M}=0.27, \Omega_{\Lambda}=0.73, H_{0}=71 \mathrm{~km} \mathrm{~s}^{-1}$ $\mathrm{Mpc}^{-1}$ cosmology is adopted throughout this paper.

\section{OPTICAL AND ULTRAVIOLET LUMINOSITY DENSITY AT $z \sim 6$}

Deep Spitzer imaging data which detect the rest-frame visible emission of $z>6$ galaxies have yielded the first constraints on the stellar mass density at $z \sim 6$. Yan et al. (2006) and Eyles et al. (2005) measured the IRAC photometry of LBGs selected using the $i$-dropout technique in the Great Observatories Origins Deep Survey (GOODS) fields. By fitting the multiband photometry of each galaxy with population synthesis models and deriving the stellar mass of each galaxy, they find a lower limit to the stellar mass density of $(0.1-0.7) \times 10^{7} M_{\odot} \mathrm{Mpc}^{-3}$, assuming a Salpeter IMF. The value is a lower limit, since only the brightest galaxies in the UV are typically detected, while many others are blended with brighter foreground sources in the IRAC images. At lower redshifts of $z \sim 5$, Stark et al. (2007) estimate the stellar mass density to be $(0.6-1) \times 10^{7} M_{\odot} \mathrm{Mpc}^{-3}$ by combining a spectroscopic and photometric redshift sample in GOODS-S. Chary et al. (2007c) adopt an alternate technique where they fit for the stellar masses of only a spectroscopic sample of galaxies at $5<z<6.5$ to derive a lower limit to the stellar mass density of $(0.3-1.1) \times 10^{6} M_{\odot} \mathrm{Mpc}^{-3}$ at $z \sim 6$. They adopt the UV luminosity function of Bouwens et al. (2007) and make the assumption that the rest-frame UV/V-band luminosity ratios of the Spitzerundetected galaxies at these redshifts are the same as the detected galaxies. The Spitzer-detected galaxies have $\nu L_{\nu}(1500 \AA) \gtrsim 2 \times$ $10^{10} L_{\odot}$ and $\nu L_{\nu}(5500 \AA) \gtrsim 10^{10} L_{\odot}$. The median visible-to-UV luminosity ratio for the Spitzer-detected galaxies is 0.5 . They then apply a completeness correction by scaling the UV luminosity density of their spectroscopic sample to the total UV luminosity density of LBGs at $z \sim 6$ as estimated by Bouwens et al. (2007). This completeness correction is a factor of 34 , which results in a total stellar mass density of $(1-3.7) \times 10^{7} M_{\odot} \mathrm{Mpc}^{-3}$ at $z \sim 6$ for a Salpeter IMF. The corresponding UV and $V$-band luminosity densities derived by integrating down to a UV luminosity of $10^{7} \mathrm{~L}_{\odot}$ are $1.4 \times 10^{8}$ and $6.6 \times 10^{7} L_{\odot} \mathrm{Mpc}^{-3}$, respectively. We call this the "high- $V$ " case. Integrating to zero luminosity results in only a $10 \%$ upward correction to these values. These values are in excellent agreement with the stellar mass density at $z \sim 6$ obtained in the hydrodynamic simulations of Davé et al. (2006) but substantially lower than the value of $8.8 \times 10^{7} M_{\odot} \mathrm{Mpc}^{-3}$ derived by Nagamine et al. (2008).

The assumption that the visible-to-UV luminosity ratios of faint LBGs is similar to that of brighter LBGs is subject to some uncertainty. Faint LBGs are predominantly in low-mass halos. The supernova feedback from the initial burst of star formation might be sufficient to inhibit or delay further star formation. This would suggest that UV-faint galaxies have lower visible-to-UV luminosity ratios than galaxies at the bright end of the UV luminosity function. This trend between rest-frame optical-UV colors with UV luminosity appears to be seen in semianalytical models (R. Somerville et al. 2001, personal communication). To assess the dependence of the $z \sim 6$ luminosity density on the optical luminosities of faint galaxies, we assume that the $\mathrm{UV} / V$-band luminosity ratios of faint Spitzer-undetected galaxies follow the corresponding ratios seen in semianalytical models. The ratio of the visible to UV luminosity density in the models, for galaxies below the Spitzer detection threshold, is 0.28 . For the UV-bright galaxies, some of which are undetected due to blending, we adopt the UV/ $V$-band luminosity ratios of the Spitzer-detected sources which are similar to the colors of UV-bright galaxies in the semianalytical models. We find that the resulting $V$-band luminosity density under these assumptions, obtained by integrating down to $\nu L_{\nu}(1350 \AA) \sim 10^{7} L_{\odot}$, is $4.2 \times 10^{7} L_{\odot} \mathrm{Mpc}^{-3}$, which is $30 \%$ smaller than the estimate of Chary et al. $(2007 \mathrm{c})$. We call this the "low- $V$ " case.

More recently, massive, evolved stellar populations have been claimed in candidate high-redshift galaxies, which would boost the derived $V$-band luminosity densities and therefore the stellar mass densities, by factors of several (Wiklind et al. 2008; Panagia et al. 2005). However, since a significant fraction of such sources are detected at $24 \mu \mathrm{m}$, a wavelength which traces the dust emission from galaxies, the evidence is strongly against these objects being at high redshifts (Chary et al. 2007b). ${ }^{2}$ Furthermore, through mid-infrared photometric redshifts, it has been demonstrated that the best candidate among these proposed high-redshift galaxies is a dusty, infrared luminous galaxies at $z \sim 1.7$ (Chary et al. $2007 \mathrm{~b}$ ). We ignore this population of galaxies for the rest of the discussion until there is any reliable evidence that they really are at $z \gg 5$.

Typically, stellar mass densities are estimated by fitting the multiwavelength spectral energy distribution of individual galaxies with population synthesis models and integrating over the resulting stellar mass distribution. However, the stellar mass density is sensitive to the choice of the stellar mass function, which is one of the parameters we are trying to constrain. Furthermore, since we do not have multiwavelength detections of faint galaxies, the only available approach involves fitting a population synthesis model to the integrated rest-frame UV and $V$-band luminosity density estimates at $z \sim 6$ derived above.

We adopt the Starburst 99 set of models which have the Padova tracks for the thermally pulsing asymptotic giant branch stars (TP-AGBs) incorporated (Leitherer et al. 1999; Vazquez \& Leitherer 2005). The variables in the models are the slope of the stellar IMF, metallicity, age of the stellar population, and stellar mass density. We consider both 0.02 and $0.4 Z_{\odot}$ templates and single-burst models. We also convolve the templates with a model for the absorption by the Ly $\alpha$ forest (Madau 1995). The fit to the luminosity density constrains the stellar mass density, as well as the total number of ionizing photons produced over the duration of the starburst. Adopting a single-burst model yields maximal ages and stellar mass densities while the total number of ionizing photons produced is insensitive to the time history of star formation.

If we adopt a Salpeter IMF with metallicity $0.02 Z_{\odot}$, we find that the optical and UV luminosity density at $z \sim 6$ are fit by a stellar population of age $100 \mathrm{Myr}$ and with a stellar mass density of $1.6 \times 10^{7} M_{\odot} \mathrm{Mpc}^{-3}$ (Fig. 1). If instead we consider the low- $V$ case, we derive a maximal stellar age of $50 \mathrm{Myr}$ and a total stellar mass of $0.8 \times 10^{7} M_{\odot} \mathrm{Mpc}^{-3}$. The presence of extinction at $z \sim 6$ would imply that reproducing the observed optical-to-UV luminosity densities will require a bluer intrinsic stellar population, corresponding to a younger age. Since the average extinction in star-forming regions at $z \sim 6$ is unknown, we do not attempt to obtain fits that include extinction.

Changing the stellar IMF will change the derived stellar mass densities. Since we use the optical and UV luminosity densities at $z \sim 6$ as the overall constraint, and evolve a single starburst of

\footnotetext{
2 The exception is a galaxy at $z=5.554$ which might have its stellar mass estimate erroneously elevated due to the presence of $\mathrm{H} \alpha$ in the Spitzer passbands. See source ID 44 in Chary et al. (2007c) for details.
} 

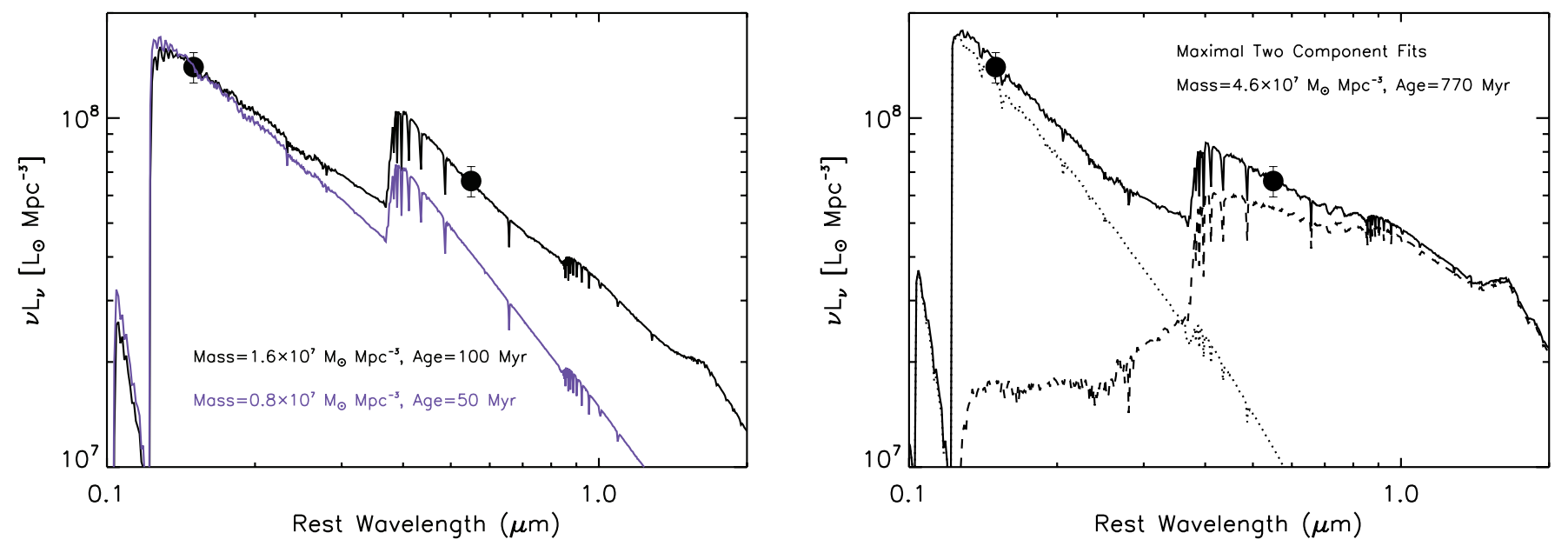

Fig. 1.-Left: Filled circles show the rest-frame 1500 and $5500 \AA$ Aluminosity density at $z \sim 6$ after appropriate completeness corrections for faint galaxies have been made. The faint galaxies have been assumed to have visible/UV luminosity ratios similar to that of $z \sim 6$ galaxies detected in Spitzer/GOODS observations (the high- $V$ case). The solid black line shows the age and stellar mass density of the template SED which fits the luminosity densities for a Salpeter stellar IMF with no dust extinction. The age indicates that if the stellar mass function had been Salpeter, the stellar population that is seen in $z \sim 6$ galaxies must have formed as late as 100 Myr earlier, i.e., at $z \sim 6.5$. If faint galaxies have lower visible-to-UV flux ratios compared to bright galaxies, the $V$-band luminosity density would decrease $30 \%$ (the low- $V$ case). The purple line represents the SED which fits the luminosity density values in this latter scenario. Right: Maximal fits to the high- $V$ luminosity density at $z \sim 6$ assuming two stellar components and a Salpeter IMF. The UV luminosity density is fit by a $10 \mathrm{Myr}$ old burst, while a $770 \mathrm{Myr}$ old, high mass-to-light ratio stellar component is the dominant contributor to the visible luminosity density.

fixed mass, the true stellar mass density is the normalization factor for the fixed mass burst. Thus, for every stellar IMF that we consider, we can derive the age, stellar mass density, and number of ionizing photons produced by requiring that the luminosity density estimates at $z \sim 6$ are reproduced. We note that the age is not a strongly constrained parameter, since we are fitting to only observations at two wavelengths and the exact age would strongly depend on the average time history of star formation. By adopting a single-burst model, we only derive maximal ages for a single stellar population.

The UV and optical luminosity densities at $z \sim 6$ are dominated by ongoing star formation and intermediate-mass stars, respectively, the latter depending on the age of the starburst. For a particular IMF, the stars with lifetimes shorter than the age of the starburst at that redshift would have presumably evolved to their end states, i.e., neutron stars or black holes. They would still be contributing to the total stellar mass density in the models, as well as the total number of ionizing photons produced by the starburst, even if they do not contribute to the UV/optical luminosities at $z \sim 6$.

An upper limit to the stellar mass density for each IMF can also be derived by adopting a two-component stellar population synthesis model (Fig. 1, right). The young stellar population, which dominates the UV luminosity density, is assumed to be $\sim 10 \mathrm{Myr}$ old. An old, high mass-to-light ratio stellar population is assumed to form in a single burst at $z \sim 20$ and evolve passively to $z \sim 6$. This implies a stellar age of $770 \mathrm{Myr}$. Naturally, a stellar IMF in which all stars have lifetimes younger than $770 \mathrm{Myr}$ will yield unphysical stellar masses and is represented by ellipses in Table 2. We note that such a two-component starburst has been considered previously by Chary et al. (2007c), who showed that the number of ionizing photons produced even in such an extreme scenario falls short of maintaining an ionized IGM, assuming a Salpeter IMF. Although concentrating all the star formation into a single $z \sim 20$ burst increases the total number of ionizing photons produced by factors of a few (see Tables 1 and 2), the recombination rate increases as $(1+z)^{3}$, which implies that a much larger number of ionizing photons are required to keep the universe ionized at these redshifts.

\section{CONSTRAINING REIONIZATION}

By fitting the measured optical and UV luminosity density at $z \sim 6$ with an evolving, single-burst model, we can determine the age of the stellar population and the stellar mass density. Integration of the number of photons produced shortward of the Lyman limit over the age of the population yields the total number of ionizing photons produced by a starburst template which fits the luminosity density values. The integral constraint has the advantage of being independent of the exact star formation history, which is likely to have a multitude of ages and $e$-folding times. Since the vast majority of ionizing photons are produced in massive stars, the single-burst model also has the advantage that the time integral of the number of ionizing photons, for starburst ages larger than $\sim 10 \mathrm{Myr}$, converges to a single value.

The number of ionizing photons produced over the history of star formation can be compared with the number of ionizing photons required to ionize the IGM. The number of photons required to initiate reionization is only 1 photon per baryon. However, to account for recombination, the production rate of ionizing photons per baryon must be $\sim 10$ times higher. To quantify the rate of ionizing photons $\dot{N}(z)$ required to keep the IGM ionized at any redshift, the rate of ionizing photons must be greater than or equal to the recombination rate.

The recombination rate $R$ is

$$
R=n_{e} n_{\mathrm{H} \text { II }} \alpha_{\mathrm{B}} C \mathrm{~s}^{-1} \mathrm{Mpc},^{-3}
$$

where $n_{e}$ is the comoving electron density, $n_{\mathrm{H}}$ is the comoving ionized hydrogen density, $\alpha_{\mathrm{B}}$ is the recombination coefficient to excited states of hydrogen, and $C$ is the ionized hydrogen clumping factor, defined as $\left\langle n_{\mathrm{H} \text { II }}^{2}\right\rangle /\left\langle n_{\mathrm{H} \mathrm{II}}\right\rangle^{2}$. Since He II reionization takes place at much lower redshifts, $z \sim 3-4$ (Sokasian et al. 2002), the comoving electron density is

$$
n_{e}=n_{\mathrm{H} \mathrm{II}}+n_{\mathrm{He} \mathrm{II}}
$$

and not $n_{\mathrm{H} \text { II }}+2 n_{\mathrm{He}}$ as has previously been adopted, although the difference is negligibly small because of the small fractional number 
TABLE 1

Stellar Mass Density at $z \sim 6$ For Different IMFs

\begin{tabular}{|c|c|c|c|c|}
\hline $\begin{array}{l}\text { Mass Range } \\
\qquad\left(M_{\odot}\right)\end{array}$ & IMF Slope $\alpha$ & $\begin{array}{l}\text { Stellar Mass Density } \\
\qquad\left(10^{6} M_{\odot} \mathrm{Mpc}^{-3}\right)\end{array}$ & $\begin{array}{l}\text { Stellar Age }{ }^{\mathrm{a}} \\
\text { (Myr) }\end{array}$ & $\begin{array}{c}\text { Ionizing Photons } \\
\text { per Baryon }^{\mathrm{b}}\end{array}$ \\
\hline 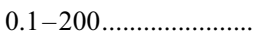 & -2.3 & 16.1 & 95 & 0.91 \\
\hline 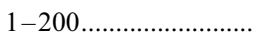 & -2.3 & 7.3 & 96 & 2.06 \\
\hline $2-200 \ldots \ldots \ldots \ldots \ldots \ldots \ldots \ldots$ & -2.3 & 6.1 & 100 & 2.69 \\
\hline 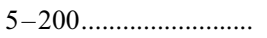 & -2.3 & 5.1 & 68 & 3.97 \\
\hline $10-200 \ldots \ldots \ldots \ldots \ldots \ldots \ldots$ & -2.3 & 2.5 & 25 & 5.50 \\
\hline 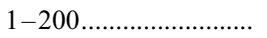 & -2.0 & 15.1 & 93 & 3.36 \\
\hline 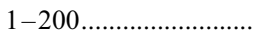 & -1.7 & 9.5 & 94 & 4.58 \\
\hline $0.5-200 \ldots \ldots \ldots \ldots \ldots \ldots \ldots$ & -2.0 & 10.7 & 94 & 2.96 \\
\hline $0.5-200 \ldots \ldots \ldots \ldots \ldots \ldots \ldots$ & -1.7 & 15.6 & 92 & 4.36 \\
\hline $1-50 \ldots \ldots \ldots \ldots \ldots \ldots \ldots \ldots$ & -2.0 & 7.0 & 95 & 1.88 \\
\hline 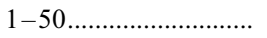 & -1.7 & 8.6 & 93 & 2.77 \\
\hline 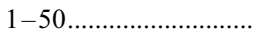 & -1.4 & 11.6 & 91 & 3.72 \\
\hline 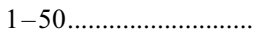 & -1.0 & 19.0 & 88 & 4.88 \\
\hline
\end{tabular}

a This is only a maximal age for a single stellar population assuming all the stars formed in a single instantaneous burst and were then allowed to passively evolve. The exact age is sensitive to the $e$-folding timescale of a starburst and cannot be derived with the data at hand.

${ }^{\mathrm{b}}$ This is the total number of ionizing photons produced over the duration of the starburst per baryon per $10^{6} M_{\odot}$ of stars formed. This needs to be multiplied by the escape fraction before a comparison with the recombination rate is made.

density of He. We adopt $\alpha_{\mathrm{B}}=2.59 \times 10^{-13}(1+z)^{3} \mathrm{~cm}^{3} \mathrm{~s}^{-1}$ for the comoving recombination coefficient (case B), assuming a gas temperature of $10^{4} \mathrm{~K}$. So, the photon production rate per baryon to balance the recombination rate at any redshift is

$$
\dot{N}(z)=\frac{R}{n_{b}} \mathrm{~s}^{-1} .
$$

The number of photons per baryon to start reionization at any redshift $N_{0}(z)$ is simply the ionized hydrogen fraction at that redshift:

$$
N_{0}(z)=\frac{n_{\mathrm{H} \text { II }}}{n_{b}} .
$$

TABLE 2

\begin{tabular}{|c|c|c|}
\hline $\begin{array}{l}\text { Mass Range } \\
\qquad\left(M_{\odot}\right)\end{array}$ & IMF Slope $\alpha$ & $\begin{array}{l}\text { Maximal Stellar Mass Density } \\
\qquad\left(10^{6} M_{\odot} \mathrm{Mpc}^{-3}\right)\end{array}$ \\
\hline $0.1-200 \ldots \ldots \ldots \ldots \ldots \ldots$ & -2.3 & 46 \\
\hline $1-200 \ldots \ldots \ldots \ldots \ldots$ & -2.3 & 21 \\
\hline $2-200 \ldots \ldots \ldots \ldots \ldots$ & -2.3 & 47 \\
\hline $5-200 \ldots \ldots \ldots \ldots \ldots \ldots$ & -2.3 & $\ldots$ \\
\hline $10-200 \ldots \ldots \ldots \ldots \ldots \ldots$ & -2.3 & $\ldots$ \\
\hline $1-200 \ldots \ldots \ldots \ldots \ldots \ldots$ & -2.0 & 36 \\
\hline $1-200 \ldots \ldots \ldots \ldots$ & -1.7 & 73 \\
\hline $0.5-200 \ldots \ldots \ldots \ldots \ldots$ & -2.0 & 39 \\
\hline $0.5-200 \ldots \ldots \ldots \ldots \ldots$ & -1.7 & 74 \\
\hline $1-50 \ldots \ldots \ldots \ldots \ldots \ldots \ldots$ & -2.0 & 26 \\
\hline $1-50 \ldots \ldots \ldots \ldots \ldots \ldots$ & -1.7 & 42 \\
\hline $1-50 \ldots \ldots \ldots \ldots \ldots$ & -1.4 & 73 \\
\hline $1-50 \ldots \ldots \ldots \ldots \ldots$ & -1.0 & 176 \\
\hline
\end{tabular}

Two-Component Stellar Mass Density at $z \sim 6$ for Different IMFs

${ }^{a}$ Maximal stellar mass density estimates at $z \sim 6$ derived using a twocomponent stellar population. The young stellar component has an age of $10 \mathrm{Myr}$ and dominates the UV luminosity density. The older stellar component has an age of $770 \mathrm{Myr}$ and dominates the stellar mass density. See the text for details.
So, the total number of ionizing photons per baryon required to maintain the increasing ionized fraction of the IGM between any redshift $z_{0}$ and redshift $z$, where $z_{0}>z$, is

$$
N(z)=N_{0}(z)+\int_{z^{\prime}=z_{0}}^{z^{\prime}=z} \dot{N}\left(z^{\prime}\right) \frac{d t}{d z^{\prime}} d z^{\prime}
$$

Reionization of hydrogen is complete by $z=6$, while reionization of helium is not. So, $N_{0}(z=6)$ is the fractional volume density of hydrogen, which is 0.93 . Equation (5) can be rewritten as

$$
\begin{aligned}
N(z=6)= & 0.93+6.475 \times 10^{-20} \\
& \times \int_{z^{\prime}=z_{0}}^{z^{\prime}=6} C \chi_{\mathrm{H} \mathrm{II}}\left(\chi_{\mathrm{H} \mathrm{II}}+\chi_{\mathrm{He} \mathrm{II}}\right)\left(1+z^{\prime}\right)^{3} \frac{d t}{d z^{\prime}} d z^{\prime} .
\end{aligned}
$$

Since $\Omega_{b} h^{2}=0.0223$, the comoving baryon volume density is 0.25 baryons $\mathrm{m}^{-3}$. Here $\chi_{\mathrm{H} \text { II }}$ and $\chi_{\mathrm{He} \mathrm{II}}$ are the volume-averaged gas fractions for ionized hydrogen and ionized helium, respectively. Care should be taken that these are derived with respect to the number density of baryons. Typical simulations provide ratios with respect to the number density of neutral hydrogen or neutral helium. In that case, we have adopted $n_{\mathrm{H}}=0.9268 n_{b}$ and $n_{\mathrm{He}}=0.073 n_{b}$.

If we neglect the small contribution to the electron density from helium, equation (6) can be simplified to

$$
\begin{aligned}
N(z=6) \approx & 0.93+6.475 \times 10^{-20} \\
& \times \int_{z^{\prime}=z_{0}}^{z^{\prime}=6} C^{\prime}\left(1+z^{\prime}\right)^{3} \frac{d t}{d z^{\prime}} d z^{\prime},
\end{aligned}
$$

where $C^{\prime}$ is $\left\langle n_{\mathrm{H}}^{2}\right\rangle /\left\langle n_{b}\right\rangle^{2}$, a quantity directly available from the Trac \& Cen (2007) simulations (see their Fig. 12). However, their estimate of $C^{\prime}$ is an average over the entire comoving volume. In practice, the clumpiness of gas which is within the sourceforming halos is already factored into the estimate of escape fraction. So, the clumping factor should be a measure of the clumpiness of 


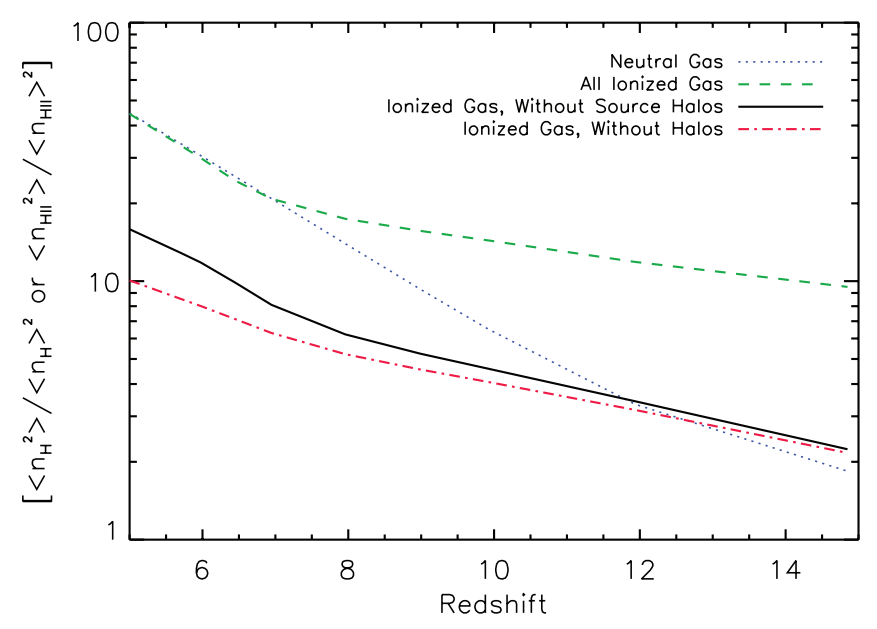

FIG. $2 a$

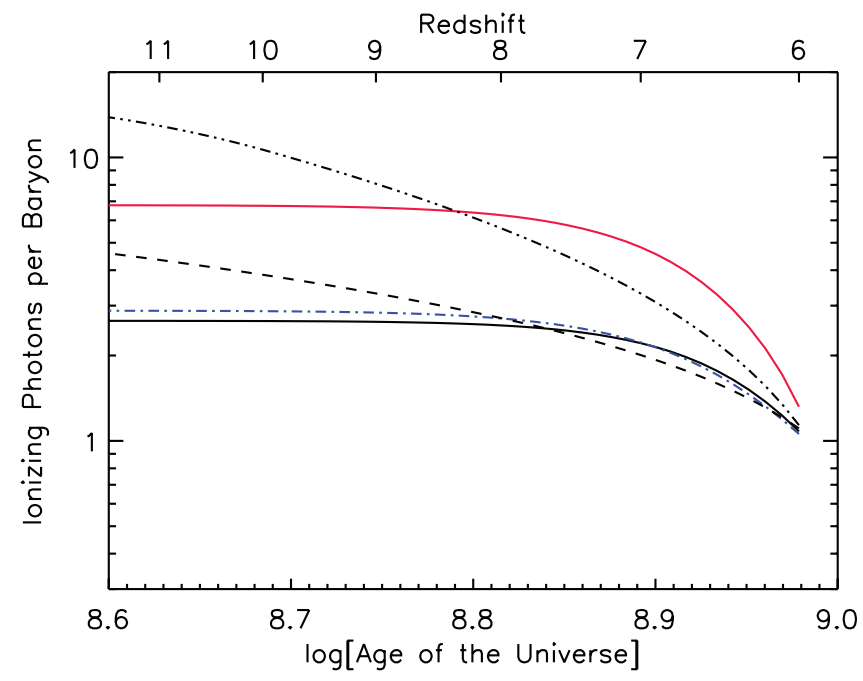

FIG. $2 b$

FIG. 2.- (a) Comparison between the clumping factors of neutral and ionized gas from the Trac \& Cen (2007) simulations for their reionization history. The blue dotted line shows the clumping of neutral gas averaged over the simulation volume. The green dashed line shows the clumping of ionized gas averaged over the simulation volume. The solid black line shows the clumping of ionized gas after star-forming halos have been excluded. The dot-dashed red line shows the clumping of ionized gas after all halos are excluded. Since the clumpiness of gas in the star-forming halos is already factored into the escape fraction, we use the solid black line as the best estimate of the clumping factor. $(b)$ Number of ionizing photons per baryon required to ionize the IGM and account for recombinations between any redshift and $z \sim 6$. The solid black line shows the number of ionizing photons per baryon assuming the reionization history and redshift-dependent clumping factor of Trac \& Cen (2007) after source halos have been excluded (solid black line in $[a]$ ). The double-dot-dashed line assumes that reionization was complete by $z \sim 12$ and the clumping factor and escape fraction remained constant at lower redshifts. The solid red line assumes the same reionization history but uses a constant clumping factor of 30 . The dot-dashed blue line assumes a constant clumping factor of 10 . The dashed line shows the number of photons derived using the parameteric solution of Madau et al. (1999) for complete hydrogen and helium reionization and the redshift-dependent clumping factor of Trac \& Cen (2007). The reionization history and properties of the IGM are clearly important in determining the number of ionizing photons required.

the IGM excluding halos with star formation in them (Fig. $2 a$ ). Therefore, we use the exact relation as shown in equation (6).

We adopt the most current estimate for the reionization history, redshift-dependent clumping factor (Fig. 2a), $n_{e}, n_{\mathrm{H} \text { II }}$, and $n_{\text {He II }}$ from the high-resolution simulations of Trac \& Cen (2007). A comparison between the number of photons per baryon from these equations compared with a similar relation derived for complete reionization by Madau et al. (1999) is shown in Figure $2 b$. The number of ionizing photons required is sensitive to the reionization history, since the recombination rate is proportional to the square of the density. If reionization is completed earlier, the recombination rate is proportionately higher than for a partially ionized medium. In that case, the number of photons per baryon needs to be higher to maintain the ionized state of the IGM as shown by the double-dot-dashed line in Figure $2 b$.

We note that the reionization history of Trac \& Cen (2007) corresponds to "late" reionization, which would result in a WMAP optical depth to Thomson scattering of $\sim 0.03$ compared to the measured value of $\tau \sim 0.09 \pm 0.03$ (Spergel et al. 2007). As characterized in Greif \& Bromm (2006) and Wyithe \& Loeb (2003), shifting the process of reionization to $z \sim 10$ would result in optical depth values which are at the high end of the observed range.

For each stellar IMF that we adopt, we obtain a library of template SEDs which are a function of the age of the starburst. We first determine the combination of age and mass which results in a best fit to the optical and UV luminosity density. This yields the stellar mass density at $z=6$ for that IMF. The range of age and mass values for a subset of IMFs considered here is shown in Table 1. As emphasized earlier, this is a maximal age for a single stellar population, since we adopt a single instantaneous burst.

The Starburst 99 model yields the number of hydrogen-ionizing photons produced as a function of age of the starburst. The escape fraction is a measure of the number of ionizing photons escaping from star-forming regions in galaxies, into the IGM. The escape fraction has been constrained to be $\sim 10 \%-15 \%$ (Siana et al. 2007; Shapley et al. 2006) in the LBG population. Thus, we adopt escape fractions of 0.1 and 0.15 . By integrating the number of ionizing photons over the derived stellar age, we can estimate the total number of photons produced over the lifetime of the evolving galaxies, multiply that with the escape fraction, and compare the result with the number of ionizing photons required to keep the IGM ionized as determined from equation (6).

\subsection{Salpeter IMF}

Figure $3 a$ shows the total number of ionizing photons produced by a starburst for a Salpeter IMF which is a power law of the form $d N / d M \propto M^{\alpha}, \alpha=-2.3$. The metallicity was $0.02 Z_{\odot}$, the escape fraction was 0.1 , and the clumping factor is shown by the solid black line in Figure $2 a$. The calculations correspond to the high- $V$ case. Different lower mass cutoffs were adopted for the Salpeter IMF as shown by the different colored lines, while the upper mass cutoff was fixed at $200 M_{\odot}$. The black line in Figure $3 a$ represents the number of ionizing photons required to keep the IGM ionized in the range $z<z^{\prime}<6$. Where the colored lines which denote the number of ionizing photons escaping from the star-forming regions cross the black line is the epoch beyond which reionization was complete.

For a Salpeter IMF extending down to $0.1 M_{\odot}$, i.e., with no low-mass cutoff, the IGM was ionized for only $60 \mathrm{Myr}$ prior to $z \sim 6$. This is an unphysically small age and indicates that if the IMF has a Salpeter shape in $z \sim 6$ galaxies, reionization must have been an extremely inhomogeneous process.

As the low-mass cutoff increases from 0.1 to $10 M_{\odot}$, the optical luminosity density produced for a fixed age for the starburst decreases. So, as the low-mass cutoff increases, the age of the starburst needs to become younger to reproduce both the UV and 

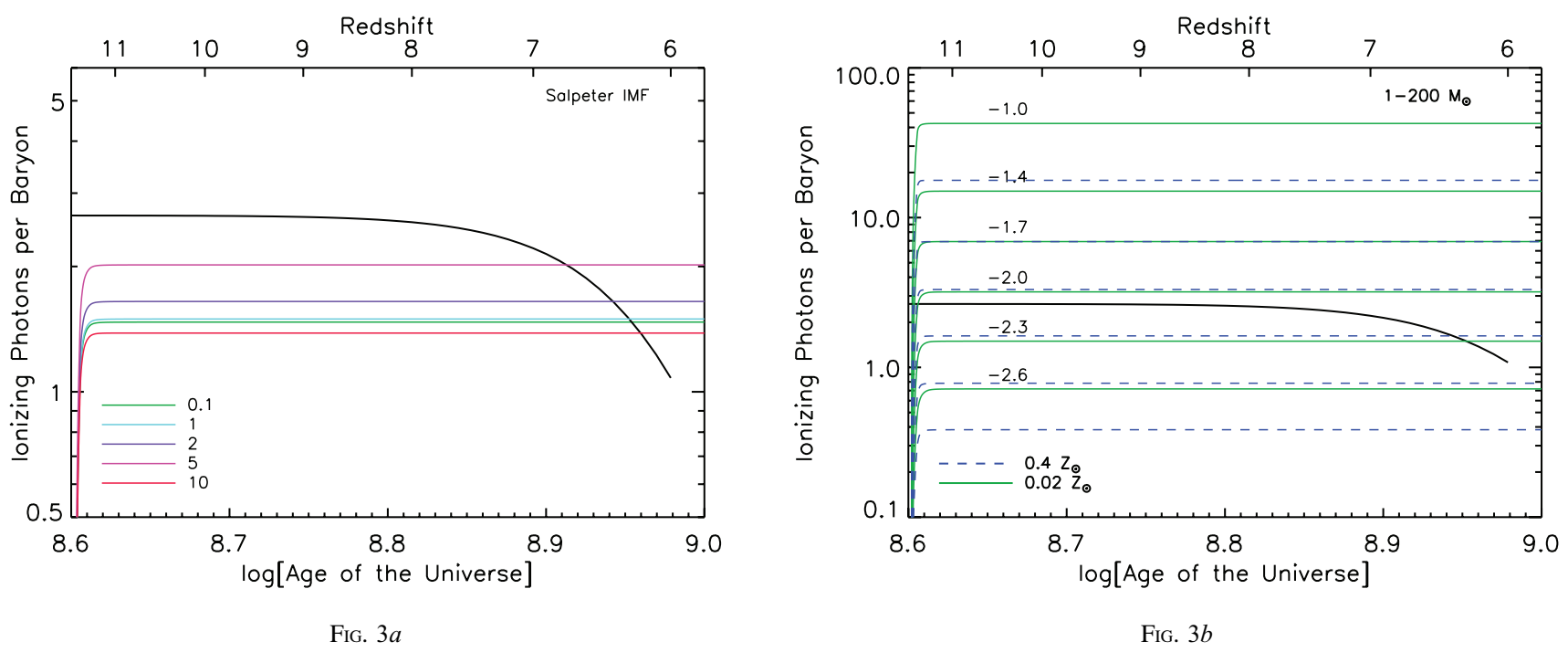

FIG. $3 b$

FIG. 3.-(a) Minimum number of ionizing photons per baryon required to ionize the IGM and account for recombinations between the plotted redshift and $z \sim 6$ (solid black line; same as in Fig. 2b). Overplotted as solid lines of different colors are the number of ionizing photons per baryon produced for a $0.02 Z_{\odot}$ Salpeter IMF $\left(d N / d M \propto M^{-2.3}\right.$ ) which reproduces the observed UV and optical luminosity density at $z \sim 6$. The different colors correspond to different low-mass cutoffs: $0.1,1,2,5$, and $10 M_{\odot}$. The stellar mass densities at $z \sim 6$ corresponding to these different IMFs are shown in Table 1. Reionization would be complete from the time at which the colored lines cross the solid black line. For the assumed clumping factor and escape fraction, a Salpeter IMF, even with a low-mass cutoff, would maintain the ionized state of the IGM only at $z<7$. (b) Number of ionizing photons for a $0.02 Z_{\odot}$ non-Salpeter IMF, with stellar masses spanning $1-200 M_{\odot}$ and different values of $\alpha$, the IMF slope (solid green line). The dashed blue line shows the number of ionizing photons for a $0.4 Z \odot$ model template with the same range of slopes. Clearly, metallicity is an important parameter in constraining the IMF.

optical luminosity density. As a result, the number of ionizing photons produced over the duration of a starburst by a Salpeter IMF with a mass cutoff at $10 M_{\odot}$ is lower than that with a mass cutoff at $0.1 M_{\odot}$.

Even in the case where the low-mass cutoff is at $5 M_{\odot}$, which produces the largest number of ionizing photons, the process of reionization has to be shorter than 140 Myr. This indicates that unless the ratio of clumping factor to escape fraction is significantly lower than what the simulations seem to indicate, a Salpeter IMF and $z_{\text {reion }}>7$ is ruled out.

The low- $V$ case corresponds to a bluer stellar population with a younger age. It would result in fewer ionizing photons over the lifetime of the starburst and make the conclusions derived above even more stringent. Thus, unless explicitly stated otherwise, our calculations below are done for the high- $V$ case.

\subsection{Modifications to the High-Redshift Stellar IMF}

Motivated by the $W M A P$ results which suggest the possibility of reionization as early as $z_{\text {reion }} \sim 11$, we investigate a range of IMFs which would produce sufficient ionizing photons to keep the IGM ionized at $z \gg 7$.

As shown in Figure $3 a$, changing the lower mass cutoff of a Salpeter IMF cannot increase $z_{\text {reion }}$ above a redshift of 7. It appears necessary to change the value of the IMF slope $\alpha$. The flatter the IMF slope, the larger the contribution of massive stars. Since most of the ionizing photons are produced by massive stars, even a small increase in the slope results in a significant increase in the number of ionizing photons produced. Changing the low-mass cutoff does not change the number of ionizing photons substantially except for the renormalization by the total mass in the IMF. If $\alpha=-2$, the IMF has equal mass in logarithmic mass bins. So for a value of $\alpha \gtrsim-2, z_{\text {reion }}$ is relatively insensitive to a change in the low-mass cutoff in the IMF.

Figure $3 b$ shows the number of ionizing photons produced by an IMF which extends from 1 to $200 M_{\odot}$ and has a range of slopes $\alpha$. For $\alpha=-2.3, z_{\text {reion }}$ falls in the range $6<z<7$, while for $\alpha=-2.0$ sufficient ionizing photons are produced to initiate reionization at $z \sim 11$ and complete the reionization process by $z \sim 6$. By comparing Figures $3 b$ and 4 , it can be seen that $z_{\text {reion }}$ for $\alpha \gtrsim-2$ is insensitive to the low-mass cutoff of the IMF. The resulting stellar mass densities at $z \sim 6$ for the IMFs considered here are shown in Table 1.

If the formation of high-mass stars is suppressed, the number of ionizing photons produced per baryon is reduced. If the cutoff is at $50 M_{\odot}$ instead of $200 M_{\odot}$, the net effect is to increase the value of $\alpha$. For example, a stellar IMF with a mass range of $1-50 M_{\odot}, \alpha=-1.7$, produces a similar number of ionizing photons as an IMF extending between 1 and $200 M_{\odot}, \alpha=-2.1$.

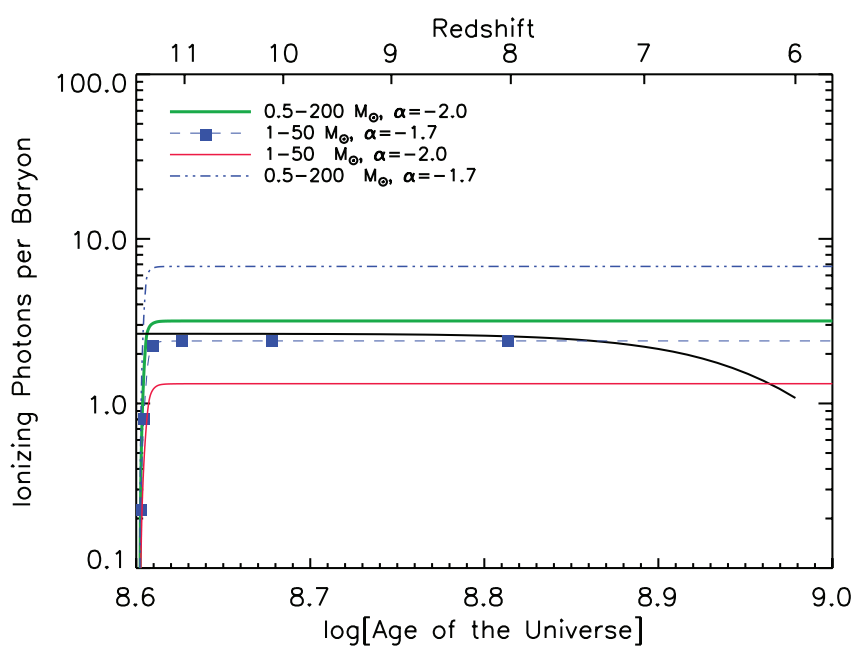

FIG. 4.-Dependence of stellar IMF on number of ionizing photons produced, as a function of IMF parameters for the reionization history of Trac \& Cen (2007). For the "late" reionization history considered by Trac \& Cen (2007), either the ratio of clumping factor to escape fraction must be much lower than the best estimates or the stellar IMF needs to have a slope index of $\alpha \sim-2.1$ if the IMF extends up to $200 M_{\odot}$. If the IMF has a high-mass cutoff at $\sim 50 M_{\odot}$, the slope needs to be $\alpha \sim-1.65$. In either scenario, this is substantially flatter than a Salpeter slope $(\alpha=-2.3)$. 


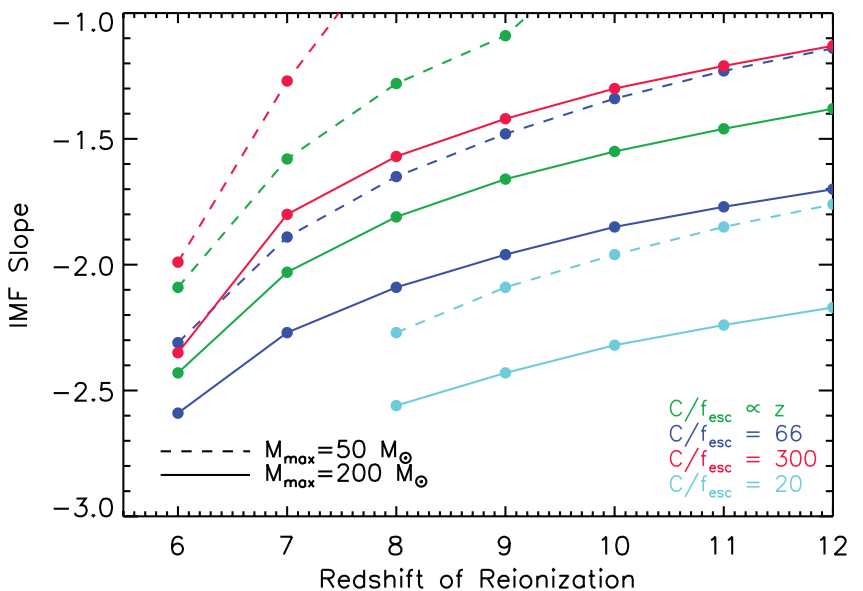

FIG. 5.-Dependence between redshift of reionization and slope of the stellar IMF for the high- $V$ case. The solid lines show the case where the IMF extends between 1 and $200 M_{\odot}$, while the dashed lines have the IMF extending between 1 and $50 M_{\odot}$. Four different values of $C / f_{\text {esc }}$ are shown. The solid green lines are our best estimate which assumes the redshift-dependent clumping factor derived by the simulations of Trac \& Cen (2007) and an escape fraction of 0.1 . The blue lines assume a redshift-independent clumping factor of 10 and an escape fraction of 0.15 . The red lines assume a constant clumping factor of 30 and escape fraction of 0.1 , while the cyan lines are an extreme case assuming a clumping factor of 10 and an escape fraction of 0.5 . For our best estimate of the clumping factor and escape fraction, the IMF must have a slope $d N / d M \propto M^{-1.65}$ for reionization to be complete by $z \sim 9$ and $d N / d M \propto M^{-1.5}$ for reionization to be complete by $z \sim 11$.

The choice of metallicity in the models is also an important parameter. For the calculations we present, the results are based on the $0.02 Z_{\odot}$ metallicity models which are the lowest available metallicity in Starburst99, although we also evaluated the results for the $0.4 Z_{\odot}$ metallicity templates as illustrated in Figure $3 b$. Lower metallicity models produce $\sim 2-3$ times as many ionizing photons as higher metallicity models (Fig. $3 b$ ). However, we note that the number of ionizing photons produced for zerometallicity single-burst templates which have normal IMFs as presented by Schaerer (2003) are very similar to the number of ionizing photons produced for $0.02 Z_{\odot}$ metallicity templates to within $\sim 10 \%-20 \%$. This implies that a Salpeter IMF, even with lower metallicity templates than our adopted ones, cannot produce sufficient ionizing photons. Thus, although the exact slope of the derived IMF is sensitive to the metallicity of the gas collapsing into the stars, the fact that the metallicity enrichment from massive stars is a relatively quick process and the metallicity of GRB hosts at $z>5$, which are presumably starforming galaxies, appears to be $\sim 0.01-0.1 Z_{\odot}$, leads us to conclude that the choice of $0.02 Z_{\odot}$ metallicity templates is a reasonable approximation.

\subsection{Dependence between Stellar IMF and $z_{\text {reion }}$}

We have generated template SEDs for a range of stellar IMF slopes and stellar mass ranges which reproduce the observed optical and UV luminosity density at $z \sim 6$. We consider two cases, one in which the stellar IMF extends between 1 and $200 M_{\odot}$ and a second in which the mass range is $1-50 M_{\odot}$. As emphasized earlier, the choice of cutoff at the low-mass end is insensitive to the result once $\alpha>-2$. We assume that the metallicity is $0.02 Z_{\odot}$ in either case. We consider a range of $-1>\alpha>-2.6$ and calculate the number of ionizing photons produced for each scenario. We then calculate the redshift at which the number of ionizing photons from the stellar population exceed the minimum required to maintain the ionized state of hydrogen. It is important to note

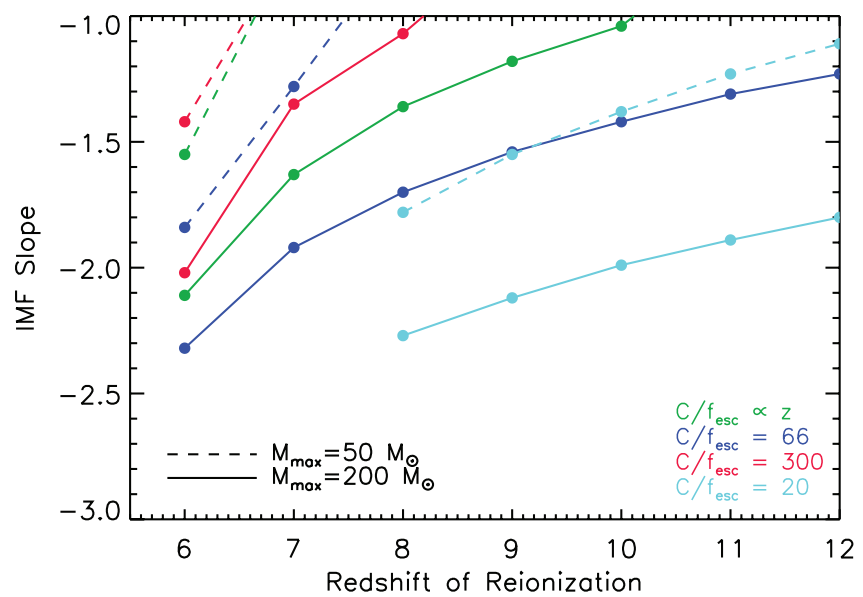

FIG. 6.- Similar to Fig. 5 but for the low- $V$ case, where the $V$-band luminosity density is $30 \%$ lower, as shown in Fig. 1 . Since a lower $V$-band luminosity density implies that fewer stars have been produced, the slope of the stellar IMF has to be even flatter, resulting in even more high-mass stars compared to Fig. 5.

that this is not simply a comparison with the solid black lines in Figures 3 and 4 which assume the particular reionization history (and thereby clumping factor) from the simulations of Trac \& Cen (2007).

If reionization were complete at higher redshifts, the recombination rate between the end of reionization and $z \sim 6$ would be higher because of the larger values of $n_{e}$ and $n_{\mathrm{H} \text { II }}$. To estimate this, we simply shift the reionization history and clumping factors of Trac \& Cen (2007) to higher redshifts and assume that the clumping factors remained constant once the IGM was completely ionized. The number of ionizing photons per baryon in the scenario where reionization is complete by $z \sim 11$ is plotted as the double-dot-dashed line in Figure $2 b$. We note that this is a factor of $\sim 4$ higher than the "late" reionization history which the models of Trac \& Cen (2007) provide. Furthermore, even if the clumping factor were to increase after the IGM was completely reionized, this would only increase the minimum number of photons per baryon required. Thus, our estimates are, at worst, a lower limit to the number of photons per baryon required to maintain the ionized state of the IGM.

We find that as the redshift of reionization increases, the value of $\alpha$ needs to increase (Fig. 5). This is not surprising, since a higher $z_{\text {reion }}$ implies a larger number of ionizing photons, which are preferentially produced in more massive stars. We also find that as the high-mass end of the stellar IMF is cut, from 200 to $50 M_{\odot}$, the slope $\alpha$ needs to increase such that a larger fraction of massive stars make up the shortfall in ionizing photons.

The relation between $z_{\text {reion }}$ and IMF slope is also sensitive to the ratio of the clumping factor to the escape fraction. Reduction in the clumping factor decreases the number of ionizing photons required, since the recombination rate is reduced. Similarly, a large escape fraction implies that a larger number of photons escape star-forming regions. So, a fewer number of ionizing photons need to be produced to reionize the IGM. As a result, in Figure 5 we plot the relation between $z_{\text {reion }}$ and $\alpha$ for different ratios of $C / f_{\text {esc }}$. A redshift-dependent clumping factor such as that shown in Figure $2 a$ and an escape fraction of 0.1 are shown as the green lines. The red lines show the estimates for a clumping factor of 30 and an escape fraction of 0.1 . The blue line show the estimates for a clumping factor of 10 and an escape fraction of 0.15 , while the cyan line is an extreme case with a clumping factor of 10 and an escape fraction of 0.5 . A similar comparison 
between the redshift when reionization is complete and the IMF slope is shown in Figure 6 for the low- $V$ case.

As can be seen for the high- $V$ case, with a Salpeter IMF ( $\alpha=$ -2.3 ), unless the ratio of clumping factor to escape fraction is much smaller than 60 , the stars in $z \sim 6$ galaxies cannot be responsible for reionization at $z>7$. For our best estimate of the clumping factor and escape fraction, if $z_{\text {reion }}=9$, the slope $\alpha=-1.65$, while if $z_{\text {reion }}=11, \alpha=-1.5$. In either scenario, only $0.2 \%$ of all baryons at $z \sim 6$ are processed through stars and are responsible for ionizing the IGM.

Our conclusions are therefore broadly consistent with the results of Tumlinson et al. (2004), who argue against the need for a very massive star population at high redshift. Their arguments are based on the metal abundance ratios in metal-poor Galactic halo stars. However, we disagree with them in the sense that a Salpeter IMF, even with a low-mass cutoff, is inadequate for producing sufficient ionizing photons to ionize the IGM while fitting the observed UV and optical luminosity density at $z \sim 6$ (Fig. 3).

Such a low-mass turnover in the stellar IMF may indeed be present at $z<2$, as has been argued by Davé (2008) and van Dokkum (2008). The former attempted to reconcile specific star formation rate estimates between models and observations, while the latter fit the color evolution of massive early-type cluster galaxies at $z<1$. We attempted to fit the observed luminosity densities at $z \sim 6$ with the IMF proposed by Davé (2008). We assumed that the $(1+z)^{2}$ evolution in the mass turnover of the stellar IMF extends up to $z \sim 4$, similar to the evolution proposed by van Dokkum (2008). Thus, the IMF was assumed to have a slope of -1.3 at $0.1 M_{\odot}<M<12 M_{\odot}$ and -2.3 at $200 M_{\odot}>M>$ $12 M_{\odot}$. For the high- $V$ case, we find that this IMF produces 3.25 ionizing photons per baryon, an age of the stellar population of $90 \mathrm{Myr}$, and a stellar mass of $1.2 \times 10^{7} M_{\odot} \mathrm{Mpc}^{-3}$. Unlike the Salpeter IMFs with a sharp cutoff shown in Figure 3, such an IMF can account for the "late" reionization history of Trac \& Cen (2007) and would maintain an ionized IGM at $z \lesssim 7$. However, for the low- $V$ case, this IMF would produce only 1.0 ionizing photons per baryon, resulting in a stellar age of $45 \mathrm{Myr}$ and a stellar mass of $3.7 \times 10^{6} M_{\odot} \mathrm{Mpc}^{-3}$, about a factor of 3 lower than the minimum required to maintain an ionized IGM at $z<7$.

\subsection{An Early Epoch of Reionization from Massive Stars?}

The fundamental assumption here is that the galaxies seen at $z \sim 6$ are the remnants of star formation responsible for reionization. It is plausible that the $z \sim 6$ galaxies that are seen in deep surveys actually comprise Population II stars with a Salpeter IMF which are responsible for a second late epoch of reionization. In that scenario, the earlier epochs would be entirely due to Population III stars which do not contribute to the UV and visible light luminosity density at $z \sim 6$, a scenario that has been considered previously (e.g., Cen 2003; Furlanetto \& Loeb 2005). These stars, which are more massive than $8 M_{\odot}$, evolve into black holes within $30 \mathrm{Myr}$, i.e., by $z \sim 6$. The number of ionizing photons required to keep the IGM ionized in the range $15<z<6$ is $\sim 11$ photons baryon $^{-1}$. An IMF extending between 10 and $100 M_{\odot}$, with a Salpeter slope of 2.3, provides a total of 1.38 photons per baryon for an escape fraction of $10 \%$ and a stellar mass density of $2.5 \times 10^{6} M_{\odot} \mathrm{Mpc}^{-3}$. The stellar mass density in massive Population III stars must therefore be $2 \times 10^{7} M_{\odot} \mathrm{Mpc}^{-3}$. Thus, if reionization was initiated by massive stars which evolve into neutron stars and black holes by $z \sim 6$, there must be as much mass density in these remnants as in the stars that we detect in the galaxies.

If, in a contrived scenario, the initial $z \sim 13$ epoch of reionization from Population III stars was relatively brief, lasting $\Delta z=2$ (e.g., Cen 2003), it would require that the stellar mass density in the initial burst was $\sim 10^{6} M_{\odot} \mathrm{Mpc}^{-3}$. This corresponds to $10 \%$ of baryons that are in stars at $z \sim 6$. Since the lifetime of $10 M_{\odot}$ stars is $\sim 30$ Myr while the interval $13<z<15$ spans $60 \mathrm{Myr}$, the first epoch of reionization must be relatively inhomogeneous and will depend on the exact epoch at which star formation in dark matter halos was initiated. Furthermore, the truncation of Population III star formation through feedback processes argues against them being significant contributors to reionization (Greif \& Bromm 2006). Measuring the distribution of Strömgren sphere size using high spatial resolution $\mathrm{H}$ i observations will reveal the true nature of the reionization history at $z>10$.

It should be noted that Population III star formation could potentially extend down to lower redshifts, depending on the effect of feedback on the metallicity of the star-forming environments. The rates of star formation in such stars are, however, thought to be $3 \times 10^{-4}$ of the Population II star formation rate and are inconsequential to the UV luminosity density or the comoving star formation rate density (Tornatore et al. 2007; Brook et al. 2007).

\section{CONCLUSIONS}

I have utilized existing data from deep HST and Spitzer surveys to provide a measure of the comoving luminosity density at ultraviolet and optical wavelengths at $z \sim 6$. In particular, I provide a measure of the contribution to the optical luminosity density from faint galaxies which are below the Spitzer detection limit. Even after accounting for faint galaxies, the optical luminosity density is a factor of 2-3 below the ultraviolet luminosity density. I fit the resulting luminosity density estimates with a stellar population synthesis model to determine the maximal age and stellar mass density at $z \sim 6$. Assuming a Salpeter IMF, I find that the stellar mass density at $z \sim 6$ is $1.6 \times 10^{7} M_{\odot} \mathrm{Mpc}^{-3}$ and the maximal age of a single stellar population is $\lesssim 100 \mathrm{Myr}$. By comparing the number of ionizing photons per baryon produced over the age of the starburst with the number of ionizing photons per baryon required to keep the IGM ionized, I find that reionization must have been a brief inhomogeneous process lasting $\lesssim 100 \mathrm{Myr}$ and must have been completed as late as $z<7$ if the stellar IMF had a Salpeter slope.

Motivated by $W M A P$ results which suggest an early epoch of reionization, I investigate the form of the stellar IMF if reionization was a single continuous process at redshifts higher than 6 . If the past history of star formation in $z \sim 6$ galaxies was responsible for reionization, I find that the slope of the stellar IMF has to be non-Salpeter with a slope of $\alpha=-1.65$ if $z_{\text {reion }}=9$, and $\alpha=-1.5$ if $z_{\text {reion }}=11$, for an IMF extending up to $200 M_{\odot}$. However, the exact slope is sensitive to the ratio of the clumping factor to the escape fraction, the metallicity of the stars, and the value of the visible luminosity density at $z \sim 6$.

On the other hand, the IGM could have been ionized in the range $6<z<15$ by a population of massive stars between 10 and $100 M_{\odot}$ which evolve into stellar remnants such as black holes by $z \sim 6$. Such a population would not be constrained by the rest-frame visible and ultraviolet luminosity density at $z \sim 6$. The resulting comoving mass density of remnants at $z \sim 6$ would be $2 \times 10^{7} M_{\odot} \mathrm{Mpc}^{-3}$ and would therefore be comparable to the stellar mass density that we see in $z \sim 6$ galaxies.

Finally, I consider the evolving stellar IMF that has been suggested by Davé (2008) and van Dokkum (2008) to account for the discrepancies in specific star formation rate estimates and the colors of early-type galaxies at lower redshifts, respectively. This IMF, if evolved out to $z \sim 4$, would result in a bottom-light IMF with a non-Salpeter slope at $M<12 M_{\odot}$. Such an IMF could produce sufficient ionizing photons to account for late reionization of 
the IGM at $z \lesssim 7$ only if the visible light luminosity density was at the high end of our assumed range.

I would like to acknowledge Avi Loeb for stimulating discussions and guidance. I would also like to thank Hy Trac for providing tabulated estimates of the ionized fraction and clump- ing factor from his simulations and for helpful suggestions. I am very grateful to Rachel Somerville for providing early results from her semianalytical models. This research is partially supported by the Spitzer Space Telescope Theoretical Research Program, which was provided by NASA through a contract issued by the Jet Propulsion Laboratory, California Institute of Technology.
Becker, R. H., et al. 2001, AJ, 122, 2850

Bouwens, R. J., et al. 2004, ApJ, 616, L79 2006, ApJ, 653, 53 2007, ApJ, 670, 928

Brook, C. B., et al. 2007, ApJ, 661, 10

Cen, R. 2003, ApJ, 591, 12

Chary, R., et al. 2007a, ApJ, 671, 272 2007b, ApJ, 665, 257 2007c, ApJ, submitted

Davé, R. 2008, MNRAS, 385, 147

Davé, R., Finlator, K., \& Oppenheimer, B. D. 2006, MNRAS, 370, 273

Eyles, L., Bunker, A., Stanway, E., Lacy, M., Ellis, R., \& Doherty, M. 2005, MNRAS, 364, 443

Fan, X., et al. 2001, AJ, 122, 2833 2006, ARA\&A, 44, 415

Furlanetto, S. R., \& Loeb, A. 2005, ApJ, 634, 1

Greif, T. H., \& Bromm, V. 2006, MNRAS, 373, 128

Kogut, A., et al. 2003, ApJS, 148, 161

Leitherer, C., et al. 1999, ApJS, 123, 3

\section{REFERENCES}

Madau, P. 1995, ApJ, 441, 18

Madau, P., Haardt, F., \& Rees, M. 1999, ApJ, 514, 648

Nagamine, K., et al. 2008, preprint (arXiv: 0802.0228)

Panagia, N., et al. 2005, ApJ, 633, L1

Schaerer, D. 2003, A\&A, 397, 527

Shapley, A., et al. 2006, ApJ, 651, 688

Siana, B., et al. 2007, ApJ, 668, 62

Sokasian, A., et al. 2002, MNRAS, 332, 601

Somerville, R., Primack, J. R., \& Faber, S. M. 2001, MNRAS, 320, 504

Spergel, D., et al. 2007, ApJS, 170, 377

Stark, D. P., Bunker, A. J., Ellis, R. S., Eyles, L. P., \& Lacy, M. 2007, ApJ, 659, 84

Tornatore, L., et al. 2007, MNRAS, 382, 945

Trac, H., \& Cen, R. 2007, ApJ, 671, 1

Tumlinson, J., et al. 2004, ApJ, 612, 602

van Dokkum, P. G. 2008, ApJ, 674, 29

Vazquez, G., \& Leitherer, C. 2005, ApJ, 621, 695

Wiklind, T., et al. 2008, ApJ, 676, 781

Wyithe, J. S. B., \& Loeb, A. 2003, ApJ, 586, 693

Yan, H., et al. 2006, ApJ, 651, 24 